\title{
Habilidades de pensamiento y su relación con el aprendizaje autorregulado en estudiantes de educación de una universidad pública de Lima
}

\author{
Thinking abilities and their relation to the self-regulated learning in \\ education students from a public university of Lima
}

\author{
Milagros Virginia Vicuña Rau ${ }^{1}$ \\ Centro Médico Master Salud
}

Recibido: $06-12-18$

\author{
Elena Sandra Sanjinés Arrieta ${ }^{2}$ \\ Crece Consultores E.I.R.L
}

\section{Resumen}

El propósito fundamental que guía el desarrollo de la presente investigación fue establecer si las habilidades de pensamiento se vinculan con el aprendizaje autorregulado, en estudiantes de la Facultad de Educación de una Universidad pública de Lima, cuya muestra alcanzo los 284 sujetos. Para tal efecto se utilizó un diseño no experimental de tipo correlacional. A la muestra seleccionada se le aplico el Cuestionario de Habilidades de Pensamiento de Alberto Acevedo y Marcela Carrera y el Cuestionario de Autorregulación para el aprendizaje de Juan Carlos Torre. A estos instrumentos se les determino previamente sus niveles de validez y confiabilidad a través de una prueba piloto, la cual determinó que las mismas cuentan con tales condiciones. Los análisis estadísticos realizados con el apoyo del software SPSS versión 24, indican que las hipótesis propuestas han sido respaldadas, es decir que las habilidades de pensamiento están relacionadas con el aprendizaje autorregulado en la muestra de estudio, tanto a nivel general $(\mathrm{r}=0,75)$, como en cada una de las dimensiones que componen cada una de estas variables.

Palabras clave: Habilidades de pensamiento; aprendizaje autorregulado; pensamiento crítico; rendimiento académico.

\begin{abstract}
The main purpose that guides the development of the current investigation was to establish if the thinking abilities are related to the self-regulated learning, in a sample of 284 students from the Education Faculty of a Public University of Lima. For that reason, a non-experimental design was used, of a correlational type. To the chosen sample were applied the Questionnaire of Thinking Abilities of Alberto Acevedo and Marcela Carrera, and the Questionnaire of Self-Regulation for learning of Juan Carlos Torre. These instruments had their levels of validity and reliability previously determinated through a pilot test, which showed that both
\end{abstract}

\footnotetext{
${ }^{1}$ Psicóloga del Centro Médico Master Salud. Correspondencia. E-mail: abcbelen@hotmail.com ${ }^{2}$ Profesora de Crece Consultores E.I.R.L. E-mail: esanjinesarrieta@gmail.com

(C) Los autores. Este artículo es publicado por la Revista de Investigación en Psicología de la Facultad de Psicología, Universidad Nacional Mayor de San Marcos. Este es un artículo de acceso abierto, distribuido bajo los términos de la licencia Creative Commons Atribucion - No Comercia_Compartir Igual 4.0 Internacional. (http://creativecommons.org/licenses/by-nc-sa/4.0/) que permite el uso no comercial, distribución y reproducción en cualquier medio, siempre que la obra original sea debidamente citada.
} 
of them fulfill both conditions. The statistical analyses, made using the software SPSS version 24, show that the proposed hypotheses have been validated; this means that the thinking abilities are related to the self-regulated learning in the studied sample, both at the general level $(r=0,75)$ and at each one of the dimensions that compose each one of these variables.

Keywords: Thinking abilities; Self-regulated learning; Critical Thinking; Academic Performance.

\section{INTRODUCCIÓN}

Desarrollar habilidades de pensamiento en los estudiantes de cualquier nivel educativo, es una de las mayores preocupaciones y retos a los que se enfrenta la educación, pues la velocidad de los cambios que ocurren en el mundo exigen una revisión y actualización constante del conocimiento que se posee para poder adecuarse a estos cambios. Por estas razones es que la formación de los estudiantes debe estar dirigida a desarrollar en ellos las competencias, capacidades, habilidades y las actitudes necesarias que permitan alcanzar un tipo de pensamiento crítico y creativo que puedan ser capaces de generar y adquirir conocimientos, pero también que les permita enfrentar y resolver problemas, además de generarles la posibilidad de lograr una actitud positiva respecto del aprendizaje permanente, que le permita autorregularlo a lo largo de toda la vida, Beas, Santa Cruz, Thomsen, y Utreras (2001).

El aprender a pensar es, por tanto, una condición básica para poder alcanzar los objetivos vitales que se han trazado, por lo que resulta imprescindible formarlo desde edades tempranas. Al respecto Vygotsky (1998) incide en la importancia del desarrollo del pensamiento y su ligazón necesaria con el entorno socio cultural. Es verdad que existen determinadas habilidades de pensamiento que van a actuar de manera automática frente a situaciones específicas, pero en estas circunstancias es altamente probable que el sujeto no tenga claro cómo fue que se activaron y menos como fue que hizo uso de ellas. Estos hechos no permiten que los sujetos puedan utilizar autónomamente sus habilidades de pensamiento, por lo que no pueden manejar la diversidad de opiniones que puedan existir sobre un hecho, por lo que difícilmente podrán llegar a un acuerdo conveniente.

Frente a estas circunstancias es que muchos jóvenes, en particular, renuncian a ejercer su pensamiento pues de esta manera se evitan conflictos. Para ellos basta con seguir la tendencia de la mayoría y así no tienen que pensar, ni argumentar a favor o en contra de una situación específica. Estas limitaciones se acrecientan en un sistema educativo que no permite el desarrollo del pensamiento, en tanto el estudiante solo se limita a recibir la información sin la menor posibilidad de analizarla y menos aplicarla a la realidad concreta. El sistema educativo peruano está formando alumnos totalmente pasivos y se le ha dejado a merced del ipod, el celular, la televisión y el "PlayStation", elementos estos que no permiten el ejercicio de un pensamiento crítico, creativo, libre, sobre todo de los jóvenes y niños. 
Marinetto (2003), afirma que los estudiantes que se dedican solo a memorizar conceptos, sin preocuparse siquiera, por lograr encontrar soluciones a los diferentes problemas que se le presentan, usando su capacidad de análisis y critica; no hacen otra cosa sino presentarse como alumnos que no tienen claridad sobre el manejo y uso eficiente de sus habilidades ligadas preferentemente hacia lo cognitivo, razón por la cual se torna indispensable y además urgente, que se implementen programas y actividades al desarrollo del pensamiento que los prepare para enfrentar con decisión y de manera exitosa, todos los estímulos que provienen desde las nuevas tecnologías de la información y del medio social en general de manera que puedan tomar las decisiones correctas tanto a nivel social, como cultural y personal.

A partir de lo señalado anteriormente tenemos claro que las habilidades de pensamiento se han convertido hoy en día en uno de los objetivos prioritarios a lograr en el terreno de la educación, pues a partir de ello se puede formar aprendices estratégicos, es decir alumnos que sepan autorregular su aprendizaje, que les permita aprender y seguir aprendiendo a lo largo de su vida. Un estudiante que autorregula su aprendizaje debe estar en condiciones de ubicar y determinar la información que le es útil de aquella que no lo es, al mismo tiempo debe mantenerse en constante automotivación para estudiar y cumplir sus obligaciones académicas, de manera que pueda incrementar su autovaloración y su sentido de la autoeficacia. Estos hechos le van a permitir manejar sus conocimientos y transferirlos de manera que pueda utilizarlos exitosamente en diversas circunstancias y contextos sociales y personales y no solo en las aulas de clase. De esta manera el aprendizaje y su autorregulación van a tomar una importante dimensión en el mundo de la educación Amestoy de Sánchez (1991).

Es claro que no todos, ni siquiera la mayoría de los estudiantes, logra autorregular su aprendizaje, es más y de acuerdo al último reporte de la Organización para la Cooperación y el Desarrollo Económicos (2015), en Ministerio de Educación del Perú (2017) el Perú es el país con peor rendimiento escolar de Sudamérica en matemáticas, lectura y ciencia. El informe detalla que nuestro país tiene el más alto porcentaje de estudiantes de 15 años que no alcanzan el nivel básico establecido. De acuerdo a lo reportado, en el área de matemáticas, los estudiantes peruanos lograron obtener 368 puntos de los 494 de promedio que plantea OCDE. De la misma manera, en lo que se refiere a la comprensión lectora, los estudiantes peruanos obtuvieron 384 puntos, en tanto el promedio de los países participantes fue de 496 puntos. En lo que se refiere al área de ciencias, el Perú tiene 373 puntos, mientras que el promedio de los países de la región fue de 501.

Estos resultados hacen necesario investigar para cambiar una realidad que no ayuda y que por el contrario pueden seguir generando apatía, descuido, poco interés, etc., en los estudiantes. Tomando en consideración todo lo señalado hasta aquí, la presente investigación busca resolver los siguientes problemas: 
1. ¿De qué manera se relacionan las Habilidades de pensamiento y el aprendizaje autorregulado en los estudiantes de la Facultad de Educación de una Universidad Pública de Lima?

2. ¿ De qué manera se relacionan las dimensiones de las habilidades de pensamiento y las dimensiones del aprendizaje autorregulado en los estudiantes de la Facultad de Educación de una Universidad Pública de Lima?

3. ¿Cuáles son los niveles de Habilidades de pensamiento que presentan los estudiantes de la Facultad de Educación de una Universidad Pública de Lima?

4. ¿Cuáles son los niveles de Aprendizaje autorregulado que presentan los estudiantes de la Facultad de Educación de una Universidad Pública de Lima?

\section{OBJETIVOS}

1. Establecer si las Habilidades de pensamiento se relacionan con el aprendizaje autorregulado en los estudiantes de la Facultad de Educación de una Universidad Pública de Lima.

2. Determinar si las dimensiones de las habilidades de pensamiento se relacionan con las dimensiones del aprendizaje autorregulado en los estudiantes de la Facultad de Educación de una Universidad Pública de Lima.

3. Describir los niveles de Habilidades de pensamiento que presentan los estudiantes de la Facultad de Educación de una Universidad Pública de Lima.

4. Establecer los niveles de Aprendizaje autorregulado que presentan los estudiantes de la Facultad de Educación de una Universidad Pública de Lima.

\section{HIPÓTESIS}

1. Las habilidades de pensamiento se relacionan de manera significativa con el aprendizaje autorregulado en los estudiantes de la Facultad de Educación de una Universidad Pública de Lima.

2. Las diversas dimensiones de las habilidades de pensamiento se relacionan de manera significativa con las dimensiones del aprendizaje autorregulado en los estudiantes de la Facultad de Educación de una Universidad Pública de Lima. 


\section{MÉTODOS Y RESULTADOS}

\section{Método}

Considerando las características que presenta, la presente investigación es de tipo básica. Sánchez y Reyes (2006), sostienen que la investigación básica, tiene como propósito el logro de conocimientos nuevos y el desarrollo de nuevas líneas de investigación. Desde este punto de vista la investigación básica busca que recoger información de la realidad para mejorar el repertorio científico existente. Desde este punto de vista la Investigación Básica busca incrementar sostenidamente los conocimientos teóricos, persigue la generalización de sus resultados con la perspectiva de desarrollar una teoría o modelo teórico científico basado en principios y leyes.

\section{Diseño de investigación}

Siguiendo a Bernal (2010); Hernández, Fernández y Baptista (2014), el diseño de investigación utilizado es el no experimental, correlacional en la medida de que no es posible la manipulación deliberada de variables para afectar a otras variables. En la investigación no experimental se observan los fenómenos tal y como se dan en su contexto natural, para después analizarlos.

\section{Muestra}

Siguiendo lo propuesto por Hernández, Fernández y Baptista (2014), el diseño de la muestra es probabilístico, pues del toda la población se han seleccionado 284 a partir del muestreo aleatorio. Para calcular el tamaño muestral se ha empleado la fórmula de Sierra Bravo (1994).

Tabla 1

Distribución de la muestra por sexo

\begin{tabular}{ccc}
\hline Sexo & Frecuencia & Porcentaje \\
\hline Varones & 117 & 41,2 \\
Mujeres & 167 & 58,8 \\
\hline Total & 284 & 100,0 \\
\hline
\end{tabular}

Tabla 2

Distribución de la muestra por nivel educativo

\begin{tabular}{ccc}
\hline Nivel & Frecuencia & Porcentaje \\
\hline Inicial & 48 & 16,9 \\
Primaria & 62 & 21,8 \\
Secundaria & 174 & 61,3 \\
\hline Total & 284 & 100,0 \\
\hline
\end{tabular}




\section{Instrumentos}

En el desarrollo del presente estudio se debió utilizar los instrumentos que se detallan a continuación:

- Cuestionario de Habilidades de Pensamiento de Alberto Acevedo y Marcela Carrera (2005).

- Cuestionario de Autorregulación para el aprendizaje de Juan Carlos Torre (2007).

\section{Análisis de validez y confiabilidad de los instrumentos}

Los análisis de confiabilidad realizados, utilizando el coeficiente alfa de cronbach, dieron como resultado que en el caso del Cuestionario de Habilidades de Pensamiento se obtuvo de 0,92 , mientras que en el caso del Cuestionario de Autorregulación para el aprendizaje se obtuvo un índice de 0,83 . En ambos casos los resultados muestran que los instrumentos de evaluación alcanzan importantes niveles de confiabilidad. Por otra parte, los análisis de validez realizados con el análisis factorial exploratorio, dieron como resultado que ambas pruebas alcanzan adecuados niveles de validez de constructo.

\section{RESULTADOS}

\section{Análisis Descriptivos Generales}

Los resultados que se muestran en la tabla 3 y figura 1, nos informan que los niveles de habilidades de pensamiento se ubican mayoritariamente a nivel medio, 55,6\% mientras que el nivel bajo llega al 22,9\% y finalmente el nivel alto llega a 21,5\%.

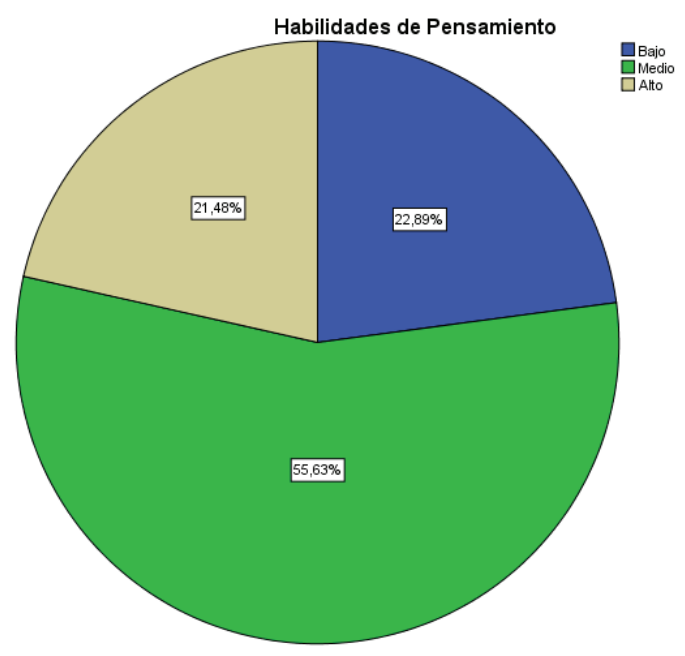

Figura 1. Niveles de habilidades de pensamiento en los estudiantes de la Facultad de Educación. 
Habilidades de pensamiento y su relación con el aprendizaje autorregulado en estudiantes de educación de una universidad pública de Lima

Tabla 3

Niveles de habilidades de pensamiento de los estudiantes

\begin{tabular}{ccc}
\hline Nivel & Frecuencia & Porcentaje \\
\hline Bajo & 65 & 22,9 \\
Medio & 158 & 55,6 \\
Alto & 61 & 21,5 \\
\hline Total & 284 & 100,0 \\
\hline
\end{tabular}

Los resultados que se muestran en la tabla 4 y figura 2 , nos informan que los niveles de aprendizaje autorregulado se ubican mayoritariamente a nivel medio, $63,00 \%$ mientras que el nivel alto llega al 21,5\% y finalmente el nivel bajo llega a $15,5 \%$.

\section{Tabla 4}

Niveles de aprendizaje autorregulado en los estudiantes

\begin{tabular}{ccc}
\hline Nivel & Frecuencia & Porcentaje \\
\hline Bajo & 44 & 15,5 \\
Medio & 179 & 63,0 \\
Alto & 61 & 21,5 \\
\hline Total & 284 & 100,0 \\
\hline
\end{tabular}

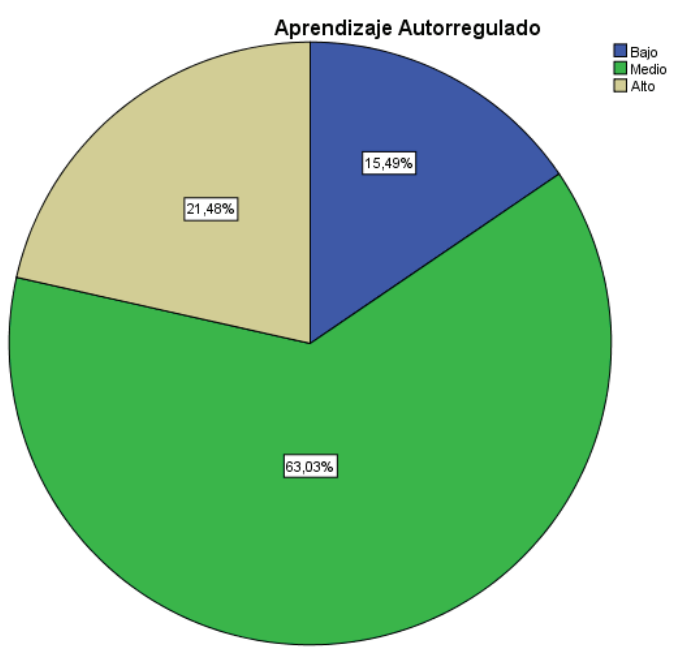

Figura 2. Niveles de aprendizaje autorregulado en los estudiantes de la Facultad de Educación.

\section{Análisis correlacionales}

Los análisis de correlación efectuados a partir de la $\mathrm{r}$ de Spearman, dan como resultado que las habilidades de pensamiento se encuentra relacionados 
significativamente con el aprendizaje autorregulado $(\mathrm{r}=0,75)$, por lo que se respalda la primera hipótesis de investigación que se planteó en la presente investigación.

\section{Tabla 5}

Análisis de Correlación (rho de Spearman) entre las habilidades de pensamiento y el aprendizaje autorregulado

\begin{tabular}{lc}
\hline Variables & Aprendizaje autorregulado \\
\hline Habilidades de pensamiento & $0,75^{* * *}$ \\
\hline
\end{tabular}

$* \mathrm{p}<, 05 * * \mathrm{p}<, 01 * * * \mathrm{p}<, 001$

$\mathrm{N}=115$

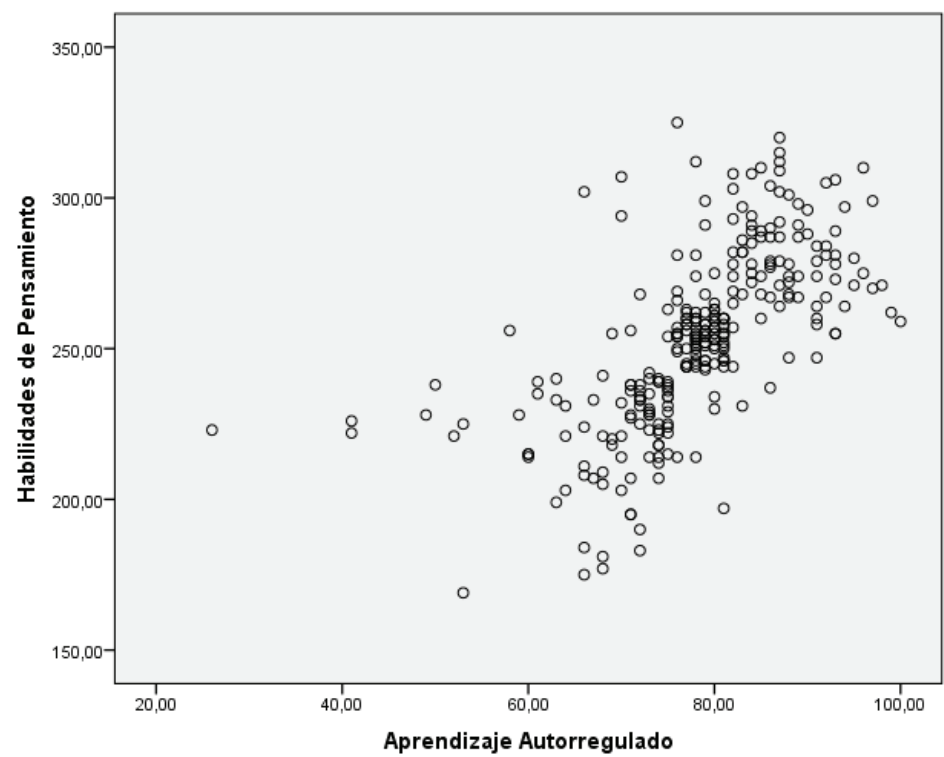

Figura 3. Correlación entre habilidades de pensamiento y aprendizaje autorregulado.

\section{Tabla 6}

Análisis de Correlación (rho de Spearman) entre las dimensiones de las habilidades de pensamiento y las dimensiones del aprendizaje autorregulado

\begin{tabular}{lcccc}
\hline Variables & $\begin{array}{c}\text { Conciencia } \\
\text { metacognitiva activa }\end{array}$ & $\begin{array}{c}\text { Control y } \\
\text { verificación }\end{array}$ & $\begin{array}{c}\text { Esfuerzo } \\
\text { diario }\end{array}$ & $\begin{array}{c}\text { Procesamiento } \\
\text { activo en clases }\end{array}$ \\
\hline Pensamiento crítico & $0,55^{* *}$ & $0,53^{* *}$ & $0,55^{* *}$ & $0,56^{* *}$ \\
Espacio y lugar de estudio & $0,45^{* *}$ & $0,41^{* *}$ & $0,46^{* *}$ & $0,50^{* *}$ \\
Uso de técnicas de estudio & $0,52^{* *}$ & $0,49^{* *}$ & $0,54^{* *}$ & $0,52^{* *}$ \\
Capacidad concentración en tareas & $0,44^{* *}$ & $0,46^{* *}$ & $0,47^{* *}$ & $0,45^{* *}$ \\
académicas & $0,56^{* *}$ & $0,54^{* *}$ & $0,53^{* *}$ & $0,51^{* *}$ \\
Motivación & & & & \\
\hline
\end{tabular}

$* \mathrm{p}<, 05 * * \mathrm{p}<, 01 * * * \mathrm{p}<, 001$

$\mathrm{N}=284$ 
Los análisis de correlación efectuados a partir de la $\mathrm{r}$ de Spearman, dan como resultado que relaciones significativas entre las dimensiones de nuestras dos variables, tal como se puede apreciar en la tabla 6 . Estos resultados respaldan a la segunda hipótesis de investigación que se planteó en la presente investigación.

\section{DISCUSIÓN}

Según los resultados estadísticos reportados respecto de la primera hipótesis, se encuentra que si existe relación estadísticamente significativas entre las habilidades de pensamiento y el Aprendizaje Este resultado es totalmente congruente con las posturas teóricas como las de Román (2004), cuando afirma que el aprendizaje autorregulado implica la utilización de un conjunto de recursos cognitivos que el estudiante utiliza con el propósito de transformar los procesos de adquisición, codificación, recuperación y regulación de la información propia de la educación formal básica, por lo que debe estar relacionada con aquellas procesos como el pensamiento y sus diversas habilidades, que van a permitirle al estudiante volverse un estudiante independiente, en la medida de que este tipo de estudiantes requieren de un buen nivel de procesamiento de la información para poder aplicarlo en sus necesidades de desarrollo y lograr nuevos aprendizajes y de manera independiente.

En este contexto los sistemas educativos cumplen un papel de primer orden puesto que su finalidad básica, está orientada a formar a los estudiantes en saberes, habilidades y valores, sin embargo la realidad nos dice que tal finalidad no se está cumpliendo. Es por esta razón que los sistemas educativos deben ser revisados y a profundidad en todas sus áreas de acción; desde los métodos de enseñanza hasta las formas de generar conocimiento y/o acceder al mismo, tal como lo plantean Leiva, Valdés, y Sepúlveda (2012). Bajo estas consideraciones, enseñar a pensar al estudiante es uno de los retos no resueltos por la Educación. Cambiar esta situación implica utilizar metodologías que involucren al estudiante a desarrollar al máximo su capacidad intelectual, con el fin de favorecer las habilidades de pensamiento y el logro de aprendizajes significativos.

Sin duda que un manejo correcto de las habilidades de pensamiento mejora significativamente la comprensión y la propia capacidad de razonar de los estudiantes lo que les permite, a su vez, enlazar los conocimientos y resolver cualquier tarea que se le pueda encomendar o simplemente dar solución a un problema. Por estas razones es que Santrock (2006), plantea que "el pensamiento implica manipular y transformar información en la memoria. Con frecuencia esto se hace para formar conceptos, razonar, pensar de manera crítica, tomar decisiones, pensar de manera creativa y resolver problemas", (p. 287).

En este camino es que Montoya (2004), afirmaba que las habilidades de pensamiento están directamente vinculadas con las capacidades cognitivas, entendidas estas como las habilidades para procesar la información derivada de los 
ejercicios de percepción, del conocimiento que ha sido previamente interiorizado y de las características personales de los sujetos que le dan un valor especifico a dicha información. Todo esto lleva a la necesaria conclusión de que las habilidades de pensamiento están orientadas a conocer, pero también a recopilar y organizar y posteriormente utilizar el conocimiento, por lo que su vinculación con procesos cognitivos como la percepción, memoria y el propio lenguaje y aprendizaje, es bastante estrecha.

\section{CONCLUSIONES}

1. Las Habilidades de pensamiento se relacionan directay significativamente con el aprendizaje autorregulado en los estudiantes de la Facultad de Educación de una Universidad Pública de Lima.

2. Las dimensiones de las Habilidades de pensamiento están vinculadas significativamente con las dimensiones del aprendizaje autorregulado en los estudiantes de la Facultad de Educación de una Universidad Pública de Lima.

3. Los niveles de habilidades de pensamiento, se ubican mayoritariamente a nivel medio, 55,6\% mientras que el nivel bajo llega al 22,9\% y finalmente el nivel alto llega a $21,5 \%$.

4. Los niveles de aprendizaje autorregulado, se ubican mayoritariamente a nivel medio, $63,0 \%$ mientras que el nivel alto llega al $21,5 \%$ y finalmente el nivel bajo llega a $15,5 \%$.

\section{REFERENCIAS}

Acevedo, A. y Carrera, M. (2005). Cuestionario de Habilidades de Pensamiento. En Alvarado, F. (2005). Relación entre habilidades de pensamiento y estrategias de aprendizaje en estudiantes de la Facultad de Educación de la Universidad de Ciencias y Humanidades. Tesis para optar el Grado Académico de Magíster en Docencia Universitaria. Universidad Nacional De Educación Enrique Guzmán Valle. Lima.

Amestoy de Sánchez, M. (1991). Desarrollo de habilidades del pensamiento. Creatividad. México: Trillas.

Beas, J., Santa Cruz, J., Thomsen, P. y Utreras, S. (2001). Enseñar a pensar para aprender mejor. Santiago: Ediciones Pontificia Universidad Católica de Chile.

Bernal, C. (2010). Metodología de la investigación. Editorial Pearson Educación. Bogotá.

Hernández, R.; Fernández, C. y Baptista, P. (2014). Metodología de la Investigación. México. Mc Graw Hill.

Leiva, J., Valdés, P., y Sepúlveda, M. (2012). Comportamiento de los estudiantes en un entorno virtual (EVA) para la enseñanza de matemática universitaria. En J. Sánchez 
Habilidades de pensamiento y su relación con el aprendizaje autorregulado en estudiantes de educación de una universidad pública de Lima

(Ed.), Memoria del XVII Congreso Internacional de Informática Educativa, TISE, (págs. 9-16). Santiago.

Marinetto, M. (2003). "Who wants to be an active citizen? The politics and practice of community involvement." Sociology the Journal of the British Sociological Association.

Ministerio de Educación del Perú (2017). El Perú en Pisa 2015, Informe Nacional de Resultados. Lima: MINEDU.

Montoya, L. (2004). Propuesta de un proceso educativo de habilidades del pensamiento como estrategias de aprendizaje en las organizaciones. México: UNAM.

Román, J. (2004). Procedimiento de aprendizaje autorregulado para universitarios: "La estrategia de lectura significativa de textos". Revista Electrónica de Investigación Psico educativa, 2(1), 113-132.

Sánchez, H. y Reyes, C. (2006). Metodología y diseño de la investigación científica. Lima: Editorial Visión Universitaria.

Santrock, J. (2006). Psicología de la educación. Segunda edición. México: McGraw-Hill.

Sierra Bravo R. (1994).Técnicas de investigación Social Teoría y ejercicios. Décima edición. Madrid: Editorial Paraninfo.

Torre Puente, J.C. (2007). Una triple alianza para un aprendizaje universitario de calidad. Madrid: Universidad Pontificia de Comillas.

Vygotsky, L. S. (1998). Pensamiento y lenguaje. Buenos Aires; Pléyade. 
